\title{
Safety risk assessment and prevention and control measures for reloading airdrop missions
}

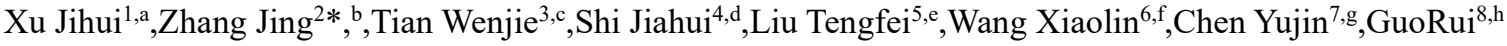 \\ ${ }^{1}$ Equipment Management and Unmanned Aerial Vehicle Engineering College Air Force Engineering University Xi'an,China \\ ${ }^{2}$ Equipment Management and Unmanned Aerial Vehicle Engineering College Air Force Engineering University Xi'an,China \\ ${ }^{3}$ Equipment Management and Unmanned Aerial Vehicle Engineering College Air Force Engineering University Xi'an,China \\ ${ }^{4}$ Equipment Management and Unmanned Aerial Vehicle Engineering College Air Force Engineering University Xi'an,China \\ ${ }^{5}$ Equipment Management and Unmanned Aerial Vehicle Engineering College Air Force Engineering University Xi'an,China \\ ${ }^{6}$ Equipment Management and Unmanned Aerial Vehicle Engineering College Air Force Engineering University Xi'an,China \\ ${ }^{7}$ Equipment Management and Unmanned Aerial Vehicle Engineering College Air Force Engineering University Xi'an,China \\ ${ }^{8}$ Equipment Management and Unmanned Aerial Vehicle Engineering College Air Force Engineering University Xi'an,China
}

\begin{abstract}
In recent years, as the heavy equipment airdrop work continues to deepen, Chinese heavy equipment airdrop business has shown a vigorous development trend, and mission training is getting closer and closer to actual combat. From the perspective of task-oriented analysis of safety risks, this paper selects the reloading airdrop mission as the entry point, and conducts research on the safety risk analysis and evaluation methods of reloading airdrop missions. At the same time, it discusses the task-oriented system safety analysis and evaluation methods, in order to carry out other tasks. The task of system security research has laid the foundation[1].
\end{abstract}

\section{Introduction}

The research topic of Chinese heavy equipment airdrop began in 1967 and was first proposed by the airborne troops. In recent years, through the step-by-step exploration and hard work of aviation personnel, China has successively conquered many key technologies such as parachute opening technology and traction control technology, and initially established GJB 7789 "Requirements for Heavy Equipment Airdrop Test" and GJB 3280 "Vehicle Artillery" Airdrop Bundling Requirements" and other standards and specifications. These constitute a systematic airdrop equipment system. Chinese newest transportation platform also completed its first airdrop training on May 9, 2018, laying the foundation for further development.

This paper takes the reloading airdrop task as the research object, deeply analyzes the airdrop task process, and combines the SHEL model to carry out the system security analysis for the airdrop task. Based on the results of the questionnaire survey, the fuzzy comprehensive evaluation based on the entropy method is used for safety assessment, and the indicators with higher risks are found. At last, the precautions are given for the conclusions of the fuzzy comprehensive evaluation.

\section{Safety risk assessment of reloading airdrop mission}

Through the analysis of the safety risk of the airdrop process, it can be found that there are many factors that lead to the failure of the reinstallation airdrop mission[2]. In addition to the four aspects of personnel, hardware, software, and environment analyzed in the SHEL model, the mission itself has problems[3]. Has a certain influence on the completion of the entire task. Based on the objective actual investigation and analysis of the questionnaire, this chapter uses MATLAB software as the calculation method to evaluate the risk of the reloading airdrop mission process through the entropy method and the fuzzy comprehensive evaluation method, and uses the MCE Fuzzy software to simulate the calculation process, aiming at the score situation, Combined with the actual work of the troops, effective prevention and control measures are given.

\subsection{Determining factor set}

According to the indicator analysis performed by the SHEL model and the indicator screening of the questionnaire survey, the set of evaluation factors is determined as shown in Figure 1:

\footnotetext{
achangan6018@163.combjij18951011529@163.com 


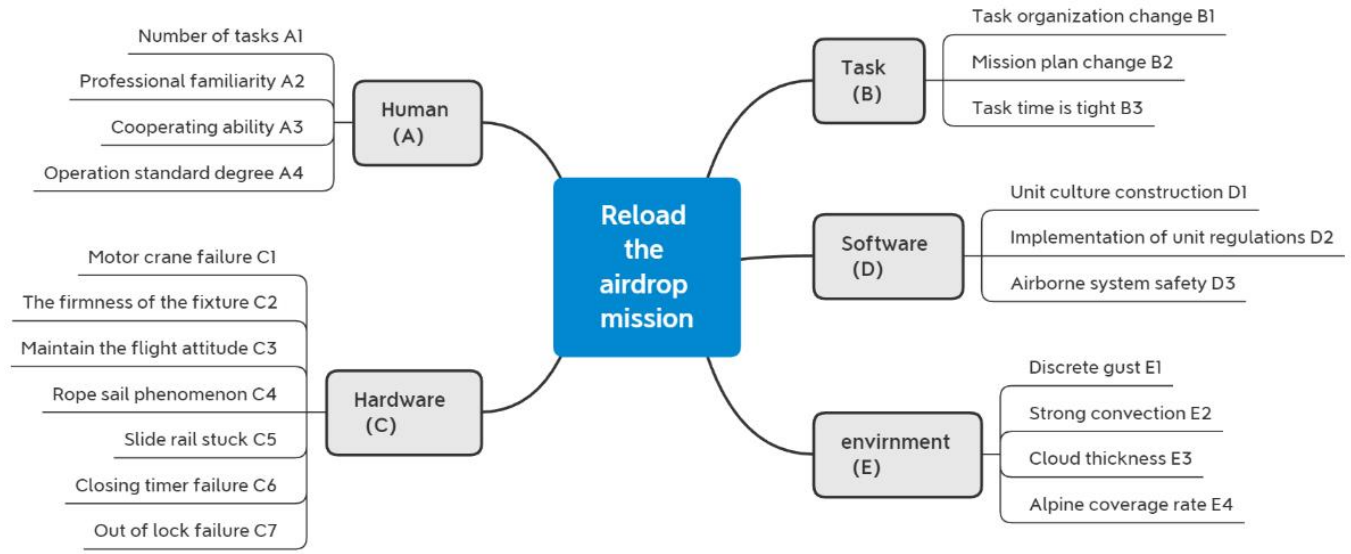

Figure 1 Factor set diagram

\subsection{Commentaries}

In this article, the risk is divided into five levels based on the situation of the reloading airdrop task, which are divided into "V1 risk is extremely high", "V2 risk is very high", "V3 risk is high", "V4 general risk", and "V5 "Low risk" represents five different levels of security, then the comment set $\mathrm{V}=\{\mathrm{V} 1$ is extremely risky, $\mathrm{V} 2$ is very risky, $\mathrm{V} 3$ is relatively risky, V4 is general risk, and V5 is low risk $\}$ [4].

Table I Weights of risk indicators for reloading airdrops

\subsection{Entropy method to establish index weight}

In order to ensure the accuracy and credibility of the weights, this article uses MATLAB software as a calculation method to sort out the answers of 22 experts in the questionnaire and objectively calculate the weights of each indicator. The obtained indicator weights are shown in Table I.

\begin{tabular}{|c|c|c|c|}
\hline First-level index & weight & Second-level index & weight \\
\hline \multirow{4}{*}{ Human factor } & \multirow{4}{*}{0.1789} & Number of tasks A1 & 0.4281 \\
\hline & & Professional familiarity A2 & 0.3643 \\
\hline & & Cooperating ability A3 & 0.1255 \\
\hline & & Operation standard degree A4 & 0.0821 \\
\hline \multirow{3}{*}{ Task factor } & \multirow{3}{*}{0.2194} & Task organization change B1 & 0.1794 \\
\hline & & Mission plan change B2 & 0.7079 \\
\hline & & Task time is tight B3 & 0.1127 \\
\hline \multirow{7}{*}{$\begin{array}{l}\text { Hardware } \\
\text { factor }\end{array}$} & \multirow{7}{*}{0.2592} & Motor crane failure $\mathrm{C} 1$ & 0.2776 \\
\hline & & The firmness of the fixture $\mathrm{C} 2$ & 0.1875 \\
\hline & & Maintain the flight attitude $\mathrm{C} 3$ & 0.0774 \\
\hline & & Rope sail phenomenon C4 & 0.1002 \\
\hline & & Slide rail stuck C5 & 0.1362 \\
\hline & & Closing timer failure $\mathrm{C} 6$ & 0.1096 \\
\hline & & Out of lock failure $\mathrm{C} 7$ & 0.1115 \\
\hline \multirow{3}{*}{ Software factor } & \multirow{3}{*}{0.2185} & Unit culture construction D1 & 0.4379 \\
\hline & & Implementation of unit regulations D2 & 0.4113 \\
\hline & & Airborne system safety D3 & 0.1507 \\
\hline \multirow{4}{*}{$\begin{array}{l}\text { envirnmental } \\
\text { factor }\end{array}$} & \multirow{4}{*}{0.1240} & Discrete gust E1 & 0.1348 \\
\hline & & Strong convection E2 & 0.2017 \\
\hline & & Cloud thickness E3 & 0.3096 \\
\hline & & Alpine coverage rate E4 & 0.3540 \\
\hline
\end{tabular}

\subsection{Construct fuzzy evaluation matrix}

This paper collects data based on the questionnaire in Chapter 3. The fuzzy relationship matrix that can be obtained is as follows:

$$
A=\left[\begin{array}{ccccc}
0.2679 & 0.3393 & 0.2857 & 0.1071 & 0 \\
0.5000 & 0.3393 & 0.1250 & 0.0357 & 0 \\
0.5000 & 0.3214 & 0.1429 & 0.0357 & 0 \\
0.5000 & 0.3750 & 0.0803 & 0.0179 & 0.0179
\end{array}\right]
$$

$$
\begin{aligned}
B & =\left[\begin{array}{llllc}
0.1607 & 0.2321 & 0.3036 & 0.2857 & 0.0179 \\
0.0893 & 0.3750 & 0.1964 & 0.3393 & 0 \\
0.1964 & 0.3571 & 0.3750 & 0.0536 & 0.0179
\end{array}\right] \\
C & =\left[\begin{array}{llllc}
0.1789 & 0.3393 & 0.2679 & 0.3143 & 0 \\
0.3214 & 0.2679 & 0.3214 & 0.0893 & 0 \\
0.3214 & 0.3929 & 0.1964 & 0.0714 & 0.0179 \\
0.4286 & 0.3214 & 0.2321 & 0.0179 & 0 \\
0.3214 & 0.3909 & 0.1786 & 0.0179 & 0 \\
0.3571 & 0.4107 & 0.1786 & 0.0536 & 0 \\
0.3571 & 0.3393 & 0.2321 & 0.0714 & 0
\end{array}\right]
\end{aligned}
$$




$$
\begin{aligned}
D & =\left[\begin{array}{llllc}
0.2321 & 0.3929 & 0.2679 & 0.1071 & 0 \\
0.4107 & 0.3036 & 0.2321 & 0.0536 & 0 \\
0.5000 & 0.3393 & 0.1071 & 0.0357 & 0.0179
\end{array}\right] \\
E & =\left[\begin{array}{llllc}
0.2982 & 0.4912 & 0.1579 & 0.0175 & 0.0351 \\
0.3509 & 0.3158 & 0.2456 & 0.0877 & 0 \\
0.1053 & 0.2982 & 0.2281 & 0.3333 & 0.0351 \\
0.1930 & 0.3684 & 0.3509 & 0.0877 & 0
\end{array}\right]
\end{aligned}
$$

\subsection{Fuzzy comprehensive evaluation}

For personnel, according to the previous text, we have known:

Weight matrix $=\left[\begin{array}{llll}0.4281 & 0.3643 & 0.1255 & 0.0821\end{array}\right]$

Fuzzy relation matrix:

$$
A=\left[\begin{array}{ccccc}
0.2679 & 0.3393 & 0.2857 & 0.1071 & 0 \\
0.5000 & 0.3393 & 0.1250 & 0.0357 & 0 \\
0.5000 & 0.3214 & 0.1429 & 0.0357 & 0 \\
0.5000 & 0.3750 & 0.0803 & 0.0179 & 0.0179
\end{array}\right]
$$

Evaluation scale matrix $=\left[\begin{array}{c}5 \\ 4 \\ 3 \\ 2 \\ 1\end{array}\right]$

MATLAB software is an advanced technical computing language used for algorithm calculation, data visualization and numerical calculation. Based on its good matrix calculation ability, the fuzzy comprehensive scores of the secondary indicators $\mathrm{A} 1, \mathrm{~A} 2, \mathrm{~A} 3$, and A4 are calculated as:

$$
\mathrm{Z}=\left[\begin{array}{l}
3.7680 \\
4.9462 \\
4.9283 \\
4.8049
\end{array}\right]
$$

The fuzzy comprehensive scores of the secondary indicators A1, A2, A3, and A4 are obtained as : 3.7689. $4.9462 、 4.9283,4.8049$. The personnel factor score obtained by $\mathrm{Z}=\mathrm{X} * \mathrm{Y}$ is 4.4280 .

In the same way, the risk scores of all secondary

\begin{tabular}{|c|c|c|c|c|c|}
\hline Total index & Score & First-level index & Score & Second-level index & Score \\
\hline \multirow{21}{*}{$\begin{array}{l}\text { Reload the } \\
\text { airdrop } \\
\text { mission }\end{array}$} & \multirow{21}{*}{3.8770} & \multirow{4}{*}{ Human factor } & \multirow{4}{*}{4.4280} & Number of tasks A1 & 3.7680 \\
\hline & & & & Professional familiarity A2 & 4.9462 \\
\hline & & & & Cooperating ability A3 & 4.9283 \\
\hline & & & & Operation standard degree A4 & 4.8049 \\
\hline & & \multirow{3}{*}{ Task factor } & \multirow{3}{*}{3.4232} & Task organization change B1 & 3.391 \\
\hline & & & & Mission plan change B2 & 3.2143 \\
\hline & & & & Task time is tight B3 & 4.7864 \\
\hline & & \multirow{7}{*}{ Hardware factor } & \multirow{7}{*}{3.9487} & Motor crane failure $\mathrm{C} 1$ & 3.6840 \\
\hline & & & & The firmness of the fixture $\mathrm{C} 2$ & 3.8214 \\
\hline & & & & Maintain the flight attitude C3 & 4.0896 \\
\hline & & & & Rope sail phenomenon $\mathrm{C} 4$ & 4.4829 \\
\hline & & & & Slide rail stuck C5 & 4.0644 \\
\hline & & & & Closing timer failure $\mathrm{C} 6$ & 4.0713 \\
\hline & & & & Out of lock failure C7 & 3.9818 \\
\hline & & \multirow{3}{*}{ Software factor } & \multirow{3}{*}{3.9598} & Unit culture construction D1 & 3.7500 \\
\hline & & & & Implementation of unit regulations D2 & 4.0714 \\
\hline & & & & Airborne system safety D3 & 4.2678 \\
\hline & & \multirow{4}{*}{ envirnmental factor } & \multirow{4}{*}{3.5894} & Discrete gust E1 & 3.9996 \\
\hline & & & & Strong convection E2 & 3.9209 \\
\hline & & & & Cloud thickness E3 & 3.1053 \\
\hline & & & & Alpine coverage rate E4 & 3.6667 \\
\hline
\end{tabular}
indicators and primary indicators are calculated as shown in Table II:

Table II Fuzzy comprehensive score

After the MATLAB calculation is completed, this article uses MCE Fuzzy to simulate the entire state process. The simulation results are roughly the same as the MATLAB calculations. It can be seen that the overall risk of reloading airdrop missions is at a higher risk stage, and relevant effective risk prevention and control must be done. Risk resolution. The simulation situation is shown in table III:

TableIII Fuzzy simulation final score map

\begin{tabular}{|c|c|c|c|c|c|}
\hline Underlying index & V1 extremely risky & V2 very risky & V3 relatively risky & V4 general risk & V5 low risk \\
\hline Closing timer failure C6 & 0.3571 & 0.4107 & 0.1786 & 0.0536 & 0 \\
\hline Out of lock failure C7 & 0.3571 & 0.3393 & 0.2321 & 0.0714 & 0 \\
\hline Unit culture construction D1 & 0.2321 & 0.3929 & 0.2679 & 0.1071 & 0 \\
\hline Implementation of unit regulations D2 & 0.4107 & 0.3036 & 0.2321 & 0.0536 & 0 \\
\hline Airborne system safety D3 & 0.5 & 0.3393 & 0.1071 & 0.0357 & 0.0179 \\
\hline Discrete gust E1 & 0.2982 & 0.4912 & 0.1579 & 0.0175 & 0.0351 \\
\hline
\end{tabular}




\begin{tabular}{|c|c|c|c|c|c|}
\hline Strong convection E2 & 0.3509 & 0.3158 & 0.2456 & 0.0877 & 0 \\
\hline Cloud thickness E3 & 0.1053 & 0.2982 & 0.2281 & 0.3333 & 0.0351 \\
\hline Alpine coverage rate E4 & 0.1930 & 0.3684 & 0.3509 & 0.0877 & 0 \\
\hline Evaluation summary & 0.2765 & 0.3457 & 0.2321 & 0.1453 & 0.0043 \\
\hline Sort & 2 & 1 & 3 & 4 & 5 \\
\hline Comment score & 5 & 4 & 3 & 2 & 1 \\
\hline \multicolumn{2}{|c|}{ Final score : 3.7564 } \\
\hline
\end{tabular}

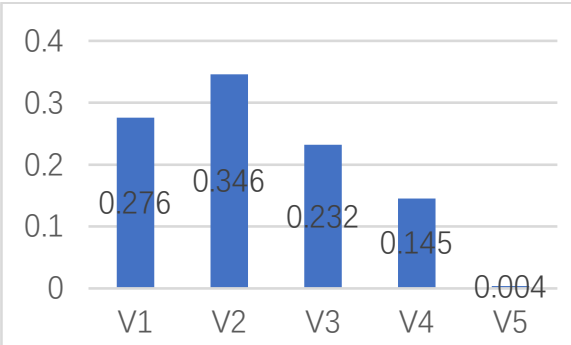

Figure 2 Fuzzy simulation membership ranking diagram

\section{Results \& Discussion}

Based on the above comprehensive assessment of the reloading airdrop task, it can be seen that the risks of personnel, software, and hardware in the airdrop process are relatively high, and unsafe accidents are prone to occur. According to the score, the risk prevention and control measures for the reloading airdrop task are sorted out. as follows:

TablelV Preventive measures

\section{Precaution}

Increase the number of training and drills and the degree of simulation The unit arranges more related professional organization training, and hires experts to guide Organize special training for team cooperation ability during training and drills

Professional technical assessment, related training, wrong setting, forgotten, omission Strengthen the team's ability to familiarize themselves with ordinary personnel, and organize multiple drills Reasonable deployment to ensure safety

Make preparations in advance to ensure that tasks can be carried out quickly Before the mission starts, check the motorized cranes as planned, and use the cranes reasonably according to regulations List equipment installation and inspection procedures

The equipment is fully secured before takeoff

The firmness of the fixture $\mathrm{C} 2$

The flight attitude remains unstable C3

\begin{tabular}{|c|c|}
\hline Rope sail phenomenon C4 & $\begin{array}{c}\text { In the design stage, use reasonable mathematical techniques to predict the occurrence of the rope sail } \\
\text { phenomenon }\end{array}$ \\
\hline Slide rail stuck C5 & Check the stuck objects in the slide rail on time, and check on time \\
\hline Closing timer failure C6 & Check the closing timer setting before taking off \\
\hline Out of lock failure C7 & Check the quality of the landing release lock before the flight \\
\hline Unit culture construction D1 & Strengthen the construction of team work style, values, team awareness, etc. \\
\hline $\begin{array}{c}\text { Implementation of unit regulations } \\
\text { D2 }\end{array}$ & Strict law enforcement, strict implementation, strict regulations \\
\hline Airborne system safety D3 & Regularly arrange software testing, and implement evaluations of related software before take-off \\
\hline Discrete gust E1 & Enhance pilot training in bad weather and avoid dangerous areas before flying \\
\hline Strong convection E2 & Try to avoid dangerous areas when flying \\
\hline Cloud thickness E3 & According to the instrument flight, try to avoid thick cloud areas \\
\hline Alpine coverage rate E4 & Try to choose a flat area \\
\hline
\end{tabular}

The next step:

1. The depth and breadth of the reloading airdrop mission process profile are greatly lacking. The next step is to comprehensively collect relevant definitions, and pay attention to real-time information and changes in related aspects, so as to have a more in-depth analysis and discussion of the entire mission process.

2 . In the evaluation model, the analysis and evaluation of factors are not specific enough. The calculation process of the evaluation method is relatively simple, and more methods should be used to combine the evaluation results to make the evaluation results more comprehensive and credible.

3. This article does not cover many influences on the quality of the cargo platform, the smoothness of the landing ground, and the flight maintenance conditions. When the conditions are unsatisfactory, the evaluation results of this paper will have large errors. How to ensure the accurate evaluation of tasks in actual combat needs further research.

\section{Conclusions}

From the perspective of task-oriented analysis of safety risks, this paper selects the reloading airdrop mission as the entry point, and conducts research on the safety risk analysis and evaluation methods of reloading airdrop missions. At the same time, it discusses the task-oriented system safety analysis and evaluation methods, in order to carry out other tasks. The task of system security research has laid the foundation.

Foundation item: National Natural Science Foundation of China (52074309) 


\section{References}

1. Tang Jianhua.Dynamic simulation calculation of airdrop system for vehicle-mounted gun[D]. Nanjing University of Science and Technology, 2016.

2. Wen Fangfang.Research on Risk Analysis and Evaluation of Real Estate Project Investment [D]. Xi'an University of Architecture and Technology, 2013.

3. Yin Shuyue, Yang Yun.Application of safety standards in military aircraft development [J].Aviation Standardization and Quality, 2010(03): 23-26.

4. Zhang Kangzhi, Li Shengxin. On the dissolution of task-based organizations and personnel [J]. Journal of Gansu Institute of Public Administration, 2007(02): 6-10.Xin Qi,Shi Zhongke.Three-dimensional nonlinear control law design for anti-crosswind anticrosswind airdrop of transport aircraft at ultra-low altitude and heavy loading[J].Acta Aeronautica Sinica, 2013, 35(7).

5. Gui Bingying.Research on Dynamic Modeling and Control Technology of Heavy Loading Airdrop [D]. Nanjing:Nanjing University of Aeronautics and Astronautics, 2012.

6. Li Chuanlin. Research on the Structural Adaptability Evaluation Method of the Task-Oriented Command Information System [D].Hunan: National University of Defense Technology,2015.GJB 7789, requirements for reloading airdrop test[S].2012.

7. GJB 3280,Binding requirements for airdrop of vehicle artillery[S].1998.

8. GJB 6854, Requirements for the airdrop of airborne equipment[S].2009.

9. GJB 7472, General technical requirements and test methods for airdrop of logistics equipment materials[S].2012.

10. DOC 9859,Safety Management Manual[S].

11. CCAR-145-R3,Civil Aircraft Maintenance Organization Certification Regulations [S].2001.

12. CCAR-25,Airworthiness Standard for Transport Aircraft[S].1995.

13. Quanqing LI ,Yingxuan L,Department S.On Constructing an Air Security System to Solve the Problem of Controlling Complex Terrain[J]. Journal of Chinese People's Armed Police Force Academy, 2016.

14. Dan C.Analysis of Security in CDMA System Airinterface $[\mathrm{J}]$.Computer knowledge and technology, 2008.

15. Li S,Liu WD, Liu ZM,et al.Discussion on Multidimensional Security System of See-Air-Land in Nuclear Power Plant[C].International Symposium on Software Reliability.Springer,Singapore,2017.

16. Xi-Tao Z,Pei W.Design and simulation of control law for large transport aircraft with heavy cargo continuous airdropping[J].flight dynamics,2013.

17. Xu B.Disturbance Observer-Based Dynamic Surface Control of Transport Aircraft With Continuous Heavy
Cargo Airdrop[J].IEEE Transactions on Systems, Man, and Cybernetics: Systems, 2016, 47(1):1-10. 